\title{
La historieta como recurso didáctico para promover el cuidado de la cavidad bucal en niños
}

\author{
Comics as a teaching resource to promote the care \\ of the oral cavity in children \\ Quadrinhos como recurso didático para promover o cuidado \\ da cavidade oral em crianças

\section{Leslie Esther Alzamora De La Rosa ${ }^{1}$ Jennifer Orozco Páez ${ }^{2}$ Jaime Enrique Plazas Román ${ }^{3}$}

Recibido: 21 de febrero de 2018

Aprobado: 15 de octubre de 2018

Publicado: 1 de julio de 2019

Cómo citar este artículo: Alzamora De la Rosa LE, Orozco Páez J, Plazas Román JE. La historieta como recurso didáctico para promover el cuidado de la cavidad bucal en niños. Rev Nac Odontol. 2019; 15(29):1-13. doi: https://doi.org/10.16925/2357-4607.2019.02.05

Artículo de investigación. https://doi.org/10.16925/2357-4607.2019.02.05

1 Corporación Universitaria Rafael Núñez, Cartagena, Colombia ORCID: https://orcid.org/0000-0002-2979-2561

Leslie Esther Alzamora De La Rosa, Programa de Odontología, Cartagena, Bolívar

Correo electrónico: leslie.alzamora@curnvirtual.edu.co

2 Corporación Universitaria Rafael Núñez, Cartagena, Colombia ORCID: https://orcid.org/0000-0003-4834-071X

3 Corporación Universitaria Rafael Núñez, Cartagena, Colombia ORCID: https://orcid.org/0000-0002-5040-6899 


\section{Resumen}

Introducción: la educación para la salud abarca una serie de herramientas de aprendizaje que ayudan a modificar las conductas relacionadas con el bienestar de las personas. La historieta es un medio de comunicación aceptado por diferentes grupos etarios, en especial por los niños y adolescentes, lo que sugiere que puede ser probada como una herramienta de enseñanza. Evaluar el uso de la historieta para promover el cuidado de la cavidad oral en pacientes pediátricos de 6 a 10 años que asisten a la escuela deportiva de la Fundación Granitos de Paz de Cartagena.

Materiales y métodos: se realizó un estudio cuasi experimental en el que se conformaron dos grupos de estudio a los que previamente se les aplicó un test sobre autocuidado de la cavidad bucal para posteriormente realizar enseñanza en salud oral, con el grupo control se utilizaron técnicas educativas basadas en charlas y demostraciones, mientras que el grupo de estudio recibió educación en salud a través de una historieta, los conocimientos adquiridos se evaluaron aplicando un test.

Resultados y conclusiones: los resultados del pretest no fueron satisfactorios para ninguno de los dos grupos de estudio. Una vez aplicadas las técnicas de educación en salud oral los resultados del postest mostraron que el $76 \%$ de los niños que fueron educados con historieta presentaron una calificación excelente, mientras que solo el $28 \%$ de los niños educados con estrategias convencionales recibieron esta valoración. Los resultados positivos ponen de manifiesto que la historieta tuvo buena aceptación y promovió la asimilación de conocimientos acerca del cuidado de la salud bucal en los niños.

Palabras clave: promoción de la salud, salud bucal, Comics como Asunto, Material de Enseñanza (DeCS).

\section{Abstract}

Introduction: Health education encompasses a series of learning tools that help modify behaviors related to people's well-being. Comics are a means of communication accepted by different age groups, especially children and adolescents, which suggests that it can be tested as a teaching tool. This work evaluates the use of comic books as a means to promote the care of the oral cavity in pediatric patients, aged 6 to 10 , who attend the sports school of the Fundación Granitos de Paz in Cartagena.

Materials and methods: A quasi-experimental study was carried out in which two study groups were formed. Both groups received a test on self-care of the oral cavity prior to receiving classes on oral hygiene. The control group used educational techniques based on lectures and demonstrations, while the study group received health education through a comic strip. The knowledge acquired was then evaluated through a test.

Results and conclusions: The results of the pretest were not satisfactory for either of the two study groups. Once the oral health education techniques were applied, the posttest results showed that $76 \%$ of children who were educated with comics received an excellent grade, while only $28 \%$ of children educated with conventional strategies received this assessment. The positive results show that the comic was well accepted and promoted the assimilation of knowledge about oral health care in children.

Keywords: health promotion, oral health, Comics as Subject, Teaching Material (DeCS).

\section{Resumo}

Introdução: A educação em saúde engloba uma série de ferramentas de aprendizagem que ajudam a modificar comportamentos relacionados ao bem-estar das pessoas. Os quadrinhos são um meio de comunicação aceito por diferentes faixas etárias, principalmente crianças e adolescentes, o que sugere que pode ser testado como uma ferramenta de ensino. Este trabalho avalia o uso de gibis como forma de promover o cuidado da cavidade oral em pacientes pediátricos, com idades entre 6 e 10 anos, que frequentam a escola de esportes da Fundação Granitos de Paz em Cartagena. 
Materiais e métodos: Foi realizado um estudo quase experimental no qual foram formados dois grupos de estudo. Ambos os grupos receberam um teste de autocuidado da cavidade oral antes de receber aulas sobre higiene bucal. 0 grupo controle utilizou técnicas educacionais baseadas em palestras e demonstrações, enquanto o grupo de estudo recebeu educação em saúde por meio de uma história em quadrinhos. 0 conhecimento adquirido foi então avaliado através de um teste.

Resultados e conclusões: Os resultados do pré-teste não foram satisfatórios para nenhum dos dois grupos de estudo. Uma vez aplicadas as técnicas de educação em saúde bucal, os resultados do pós-teste mostraram que $76 \%$ das crianças educadas com quadrinhos receberam uma nota excelente, enquanto apenas $28 \%$ das crianças educadas com estratégias convencionais receberam essa avaliação. Os resultados positivos mostram que o quadrinho foi bem aceito e promoveu a assimilação de conhecimentos sobre saúde bucal em crianças.

Palavras-chave: promoção da saúde, saúde bucal, quadrinhos como sujeito, material didático (DeCS).

\section{Introducción}

La caries dental es una patología de alta prevalencia en la población infantil. Las complicaciones como el dolor y la pérdida de estructuras dentarias, a temprana edad, afectan directamente la calidad de vida de los niños. El cuarto estudio nacional de salud bucal (ENSAB IV) realizado en Colombia, reportó que el 47,10 \% y el 62,10\% de los niños de 3 y 5 años respectivamente, han tenido experiencia de caries (1).

Estos reportes han conducido a reforzar la educación en salud oral en los niños y en sus padres o representantes legales, por esta razón, los programas de promoción en salud oral dirigen sus esfuerzos a la generación de responsabilidades en cada persona respecto a los hábitos higiénicos de la cavidad bucal, para obtener una ganancia de bienestar mediante la modificación o establecimiento de aspectos conductuales, culturales y estilos de vida que promuevan la salud oral, encaminados a la prevención de enfermedades. Así mismo, desde la odontología preventiva se vienen direccionando esfuerzos hacia la búsqueda o generación de nuevos e ingeniosos métodos que incentiven en los niños la adopción de hábitos y conductas saludables para el mantenimiento de una buena salud bucal (2-4).

Sanabria et al., mencionan que esta formación debe comenzar desde edades tempranas, por la facilidad que representa formar actitudes positivas hacia la salud en un niño que modificarlas en un adulto. Respaldados en esto, diferentes educadores en salud se valen de un conjunto de herramientas y estrategias de aprendizaje lúdico-pedagógicas para modificar en los niños conductas relacionadas con la salud (5).

John et al. (6) evaluaron el impacto de tres métodos de educación en salud diferentes en niños preescolares, un grupo de niños fue educado por un odontólogo, otro grupo por el profesor de la clase entrenado por un odontólogo y el último grupo por residentes de odontología disfrazados e imitando personajes de dibujos animados. 
El grupo educado mediante dramatizaciones mostró una mejoría significativa en la higiene bucal en comparación con los grupos educados bajo otros métodos, por lo tanto, propusieron que el drama como método de educación para la salud puede tener un mayor impacto en la actitud y las prácticas de la salud oral de los preescolares. Por su parte, Yanti et al. (7) demostraron la eficacia de videos animados como herramienta para la educación en salud oral en estudiantes sordos de 10 a 15 años, esto se evidenció en el aumento del conocimiento y la mejoría de la higiene oral en los niños incluidos en el estudio.

Como características esenciales, una herramienta de educación debe ser de bajo costo, alta cobertura y de fácil apropiación por parte de la población a la cual se dirige. El comic o historieta es uno de los medios de comunicación más aceptados, principalmente por la población infantil, combina una serie de aspectos importantes, tiene un componente lúdico, el humor y la creatividad, convirtiéndose en una buena alternativa para desarrollar el proceso enseñanza-aprendizaje (8).

Como recurso didáctico, la historieta tiene la capacidad de resumir información para quien la crea, y capta la atención de quien la lee y observa, por lo tanto, se convierte en una herramienta para transmitir información específica a una determinada población, mediante el uso de imágenes y palabras. Las historietas publicadas en periódicos, revistas u otros medios de comunicación llegaron a ser uno de los textos más leídos en los distintos países del mundo debido al abordaje cómico de su información, lo que facilita el entendimiento. Se ha convertido en objeto de estudio como herramienta de enseñanza en distintas áreas del conocimiento demostrando que la enseñanza hecha con caricaturas afecta los logros de los estudiantes y la retención de conocimientos positivamente $(9,10)$.

La implementación de la historieta como recurso didáctico representaría una oportunidad para mejorar la asimilación de conocimientos referentes al cuidado de la salud bucal en pacientes pediátricos. Teniendo en cuenta lo anteriormente expuesto, el objetivo de este estudio fue evaluar la eficacia del uso de historietas en la enseñanza del cuidado de la cavidad oral en pacientes pediátricos de 6 a 10 años que asisten a la escuela deportiva de la Fundación Granitos de Paz en Cartagena, Colombia, con el fin de minimizar la aparición de enfermedades bucales. 


\section{Materiales y métodos}

Se realizó un estudio cuasi experimental con grupo control, en el que se realizaron medidas pretest y postest. La población estuvo conformada por niños de la escuela deportiva de fútbol de la Fundación Granitos de Paz, localizada en Cartagena de Indias, Colombia, y quienes hacían parte de familias residentes en barrios con estrato socioeconómico 1 y 2. Los criterios de inclusión fueron los siguientes: niños de ambos sexos, de 6 a 10 años de edad, que en el momento del estudio no habían recibido ningún tipo de educación en salud oral, niños que supieran leer y que sus padres autorizaran la participación voluntaria en el estudio. Se excluyeron niños con dificultad en competencias lectoras y estudiantes que presentaran algún tipo de discapacidad cognitiva.

La muestra del estudio estuvo conformada por 50 niños seleccionados a través de un muestreo no probabilistico, intencional. A partir de la muestra se conformaron dos grupos de estudio: un grupo de 25 niños a quienes se les hizo enseñanza en salud oral utilizando una historieta (grupo experimental) y un grupo control conformado por 25 niños que recibieron educación en salud oral realizada a través de charlas y demostraciones con macromodelos (grupo control). En cada grupo de estudio se incluyeron cinco niños de cada edad, es decir, cinco niños de 6 años, cinco de 7 años, cinco de 8 años, cinco de 9 años y cinco de 10 años, para un total de 25 niños por grupo. Previo a su ejecución, este trabajo fue presentado y aprobado por el comité de ética institucional de la Corporación Universitaria Rafael Núñez (CURN).

\section{Diseño de la historieta}

Se diseñó una historieta orientada a desarrollar toda la información necesaria para que los niños aprendieran el cuidado de la cavidad oral de manera sencilla, práctica y llamativa. Los protagonistas corresponden a una pareja de castores con grandes dientes, encargados de enseñar los cuidados básicos de la cavidad bucal, estos son: el cepillado dental, el uso de seda dental y enjuague bucal, presentando a los implementos de higiene oral como súper héroes y a la caries dental como villano (figura 1). 

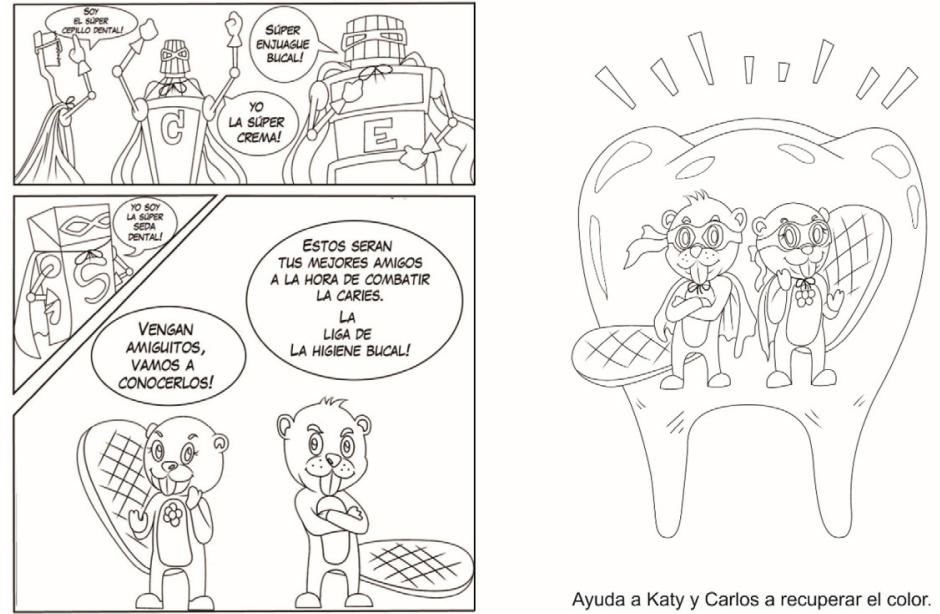

Figura 1. Segunda y tercera página de la historieta sin colorear. Los castores Katy y Carlos presentan a los implementos de higiene bucal como amigos súper héroes. El niño debe colorear para identificar a los personajes.

Fuente: elaboración propia

El contenido, la secuencia de los hechos y los dibujos animados de la historieta fueron propuestos por estudiantes de octavo semestre del programa de odontología de la CURN. El objetivo de las actividades propuestas en cada página estuvo dirigido a promover en los niños funciones como identificar, relacionar, reflexionar y aplicar lo aprendido. El boceto y la impresión de la historieta fueron realizados por un profesional en diseño gráfico (figura 2).
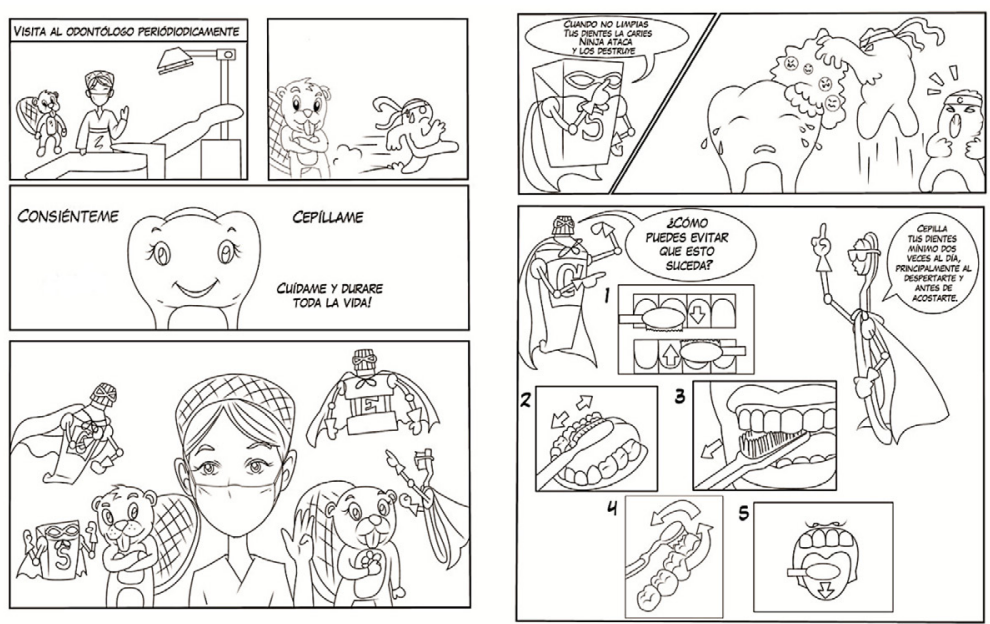

Figura 2. Cuarta y quinta página de la historieta sin colorear. El objetivo de las actividades a desarrollar en estas páginas, es que el niño logre reconocer, relacionar y aplicar las técnicas de higiene oral.

Fuente: elaboración propia 


\section{Implementación de estrategias para la enseñanza del cuidado de la salud bucal}

Antes de implementar los métodos de enseñanza en salud oral (charla-demostración o historieta), se explicó a los representantes legales y a los participantes la finalidad del estudio y sus beneficios, la participación voluntaria de los niños se confirmó mediante asentimiento informado y la firma del consentimiento informado por parte de los padres. Posterior a esto, cada niño respondió un test con el propósito de evaluar los conocimientos previos acerca de los cuidados de la salud bucal, el test constó de 10 preguntas puntuales acerca de los implementos de higiene oral y las principales medidas para el mantenimiento de la salud bucal, seguidamente se evaluó el estado de higiene bucal mediante la determinación del índice de biopelícula dental según O'Leary, se utilizó una sustancia reveladora de placa bacteriana ditonos (EUFAR).

Al grupo experimental se le hizo entrega de la historieta previamente diseñada, se le explicó a cada niño y padre o representante, el modo de uso e indicaciones para leerla y colorearla durante un mes. Se enfatizó a los padres estar atentos al desarrollo de la historieta en casa e incentivar a los niños la aplicación de las medidas de higiene oral aprendidas durante su lectura. Por otra parte, a los 25 niños del grupo control se les realizó educación en salud oral mediante una charla, utilizando imágenes y haciendo demostraciones con macromodelos de la cavidad bucal, se indicó a los niños aplicar durante un mes las medidas de higiene oral aprendidas y también se solicitó a los padres apoyo durante el proceso.

Un mes después, se realizaron visitas de control a ambos grupos de estudio para verificar el cumplimiento de los compromisos asumidos y se realizó un refuerzo de conocimientos de medidas para el cuidado de la salud oral utilizando la misma metodología implementada a cada grupo.

Dos meses después de haber realizado la enseñanza, los niños fueron citados para aplicar nuevamente el test de conocimientos a fin de comprobar el grado de asimilación de los mismos por parte de ellos a partir de los métodos de enseñanza implementados y poder establecer si un método fue más efectivo que el otro. Además, se determinó por segunda vez el índice de biopelícula dental y se verificó la utilización de las historietas.

Los datos obtenidos, correspondientes a variables sociodemográficas y resultados de índice de placa y resultados de prueba de conocimientos, fueron tabulados en una tabla de Excel y analizados mediante tablas de frecuencia y porcentaje. 


\section{Resultados}

Al analizar las variables sociodemográficas se observó que el 92 \% ( $n=46)$ estuvo representado por el sexo masculino y solo el $8 \%(n=4)$ por el sexo femenino, esto debido a que la población de estudio provino de una escuela de fútbol de una fundación, deporte que es practicado en su mayoría por hombres. Respecto a la edad, cada grupo etario estuvo constituido por igual número de participantes, 10 niños por cada grupo de edad distribuidos por conveniencia. El nivel socioeconómico estableció a 30 niños en estrato 1 lo que corresponde a un $60 \%$ de la muestra y 20 en estrato 2 un $40 \%$.

\section{Valoración del índice de O'Leary}

En el grupo control se observó que antes de la intervención 5 (20 \%) niños presentaron un índice de biopelícula alto, después de realizada la charla este número disminuyó a 2 (8 \%) participantes y 17 (68 \%) presentaron índice de biopelícula bajo. En el grupo experimental, se encontró que antes de implementar la historieta, 3 (12 \%) niños presentaron un índice de placa alto, después de implementarla, solo 1 (4 \%) niño permaneció con un índice elevado, mientras que 23 (92 \%) participantes presentaron un índice bajo. Al comparar los datos obtenidos antes y después de la intervención se observó que la disminución del índice de biopelícula para ambos grupos fue estadísticamente significativa $(p<0,05)$ (tabla 1).

Tabla 1. Comparación del índice de biopelícula en grupo control y grupo experimental, antes y después de implementar la estrategia de enseñanza en salud oral

\begin{tabular}{|c|c|c|c|c|c|c|c|}
\hline \multirow{2}{*}{ GRUPO } & \multirow[b]{2}{*}{ Ind. Biopelícula } & \multirow[t]{2}{*}{ Ind. Biopelícula } & \multicolumn{3}{|c|}{ Después de la intervención (n) } & \multirow{2}{*}{ Total } & \multirow{2}{*}{$\mathrm{p}^{\star}$} \\
\hline & & & Bajo & Medio & Alto & & \\
\hline \multirow{4}{*}{ 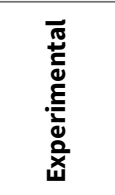 } & \multirow{3}{*}{$\begin{array}{l}\text { Antes de la } \\
\text { intervención }\end{array}$} & Bajo & 13 & 0 & 0 & 13 & \\
\hline & & Medio & 9 & 0 & 0 & 9 & $\underset{\pi}{\stackrel{1}{8}}$ \\
\hline & & Alto & 1 & 1 & 1 & 3 & $0^{-}$ \\
\hline & \multicolumn{2}{|c|}{ Total } & 23 & 1 & 1 & 25 & \\
\hline \multirow{4}{*}{$\begin{array}{l}\overline{0} \\
\stackrel{0}{2} \\
\text { Oे }\end{array}$} & \multirow{3}{*}{$\begin{array}{l}\text { Antes de la } \\
\text { intervención }\end{array}$} & Bajo & 15 & 0 & 0 & 15 & \\
\hline & & Medio & 2 & 3 & 0 & 5 & న \\
\hline & & Alto & 0 & 3 & 2 & 5 & 0 \\
\hline & \multicolumn{2}{|c|}{ Total } & 17 & 6 & 2 & 25 & \\
\hline \multirow{4}{*}{ 흄 } & \multirow{3}{*}{$\begin{array}{l}\text { Antes de la } \\
\text { intervención }\end{array}$} & Bajo & 28 & 0 & 0 & 28 & \\
\hline & & Medio & 11 & 3 & 0 & 14 & - \\
\hline & & Alto & 1 & 4 & 3 & 8 & 0 \\
\hline & \multicolumn{2}{|c|}{ Total } & 40 & 7 & 3 & 50 & \\
\hline
\end{tabular}

* Prueba de McNemar-Bowker

Fuente: elaboración propia 


\section{Evaluación de conocimientos}

El resultado del test de conocimiento realizado antes y después de la implementación de las estrategias de enseñanza en salud oral (historieta y charlas) se reportó de acuerdo al número de respuestas acertadas por los niños de la siguiente manera: malo (1 a 3 preguntas correctas), regular (4 a 6 preguntas correctas), bueno (7 a 9 preguntas correctas) y excelente (10 preguntas correctas).

El pretest reveló que todos los niños incluidos en el estudio presentaron un conocimiento escaso respecto a los cuidados que se deben tener en cuenta para mantener la salud bucal, la calificación que primó en ambos grupos de estudio fue regular. Después de realizar la intervención y aplicar el postest o test final, en el grupo control se observó que solo 7 (28 \%) niños obtuvieron un resultado excelente y, en su mayoría, 12 (48 \%) lograron un resultado bueno, mientras que en el grupo experimental, después de interactuar con la historieta, 19 (76 \%) niños obtuvieron calificación excelente, 5 (20 \%) lograron calificación bueno y solo 1 (4 \%) niño obtuvo una calificación regular. Al comparar los resultados del pre y postest se observó que existe diferencia estadísticamente significativa entre los grupos de estudio y entre los resultados antes y después de la intervención a nivel intragrupo. ( $p<0,005)$ (tabla 2).

Tabla 2. Resultados pretest y postest en los dos grupos de estudio

\begin{tabular}{|c|c|c|c|c|c|c|c|}
\hline \multirow{2}{*}{ 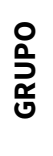 } & \multirow{2}{*}{$\begin{array}{l}\text { VARIABLE } \\
\text { Regular }\end{array}$} & & \multicolumn{3}{|c|}{$\begin{array}{l}\text { Conocimiento después de la } \\
\text { intervención }\end{array}$} & \multirow{2}{*}{ Total } & \multirow{2}{*}{ p* } \\
\hline & & & Regular & Bueno & Excelente & & \\
\hline \multirow{3}{*}{ 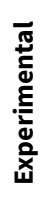 } & \multirow{2}{*}{$\begin{array}{l}\text { Conocimiento antes de la } \\
\text { intervención }\end{array}$} & Malo & 0 & 0 & 6 & 6 & \multirow{3}{*}{0,000} \\
\hline & & Regular & 1 & 5 & 13 & 19 & \\
\hline & Total & & 1 & 5 & 19 & 25 & \\
\hline \multirow{4}{*}{ 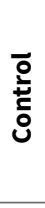 } & \multirow{3}{*}{$\begin{array}{l}\text { Conocimiento antes de la } \\
\text { intervención }\end{array}$} & Malo & 0 & 0 & 2 & 2 & \multirow{4}{*}{0,000} \\
\hline & & Regular & 3 & 12 & 5 & 20 & \\
\hline & & Bueno & 3 & 0 & 0 & 3 & \\
\hline & Total & & 6 & 12 & 7 & 25 & \\
\hline \multirow{4}{*}{ 흉 } & \multirow{3}{*}{$\begin{array}{c}\text { Conocimiento antes de la } \\
\text { intervención }\end{array}$} & Malo & 0 & 0 & 8 & 8 & \multirow{4}{*}{0,000} \\
\hline & & Regular & 4 & 17 & 18 & 39 & \\
\hline & & Bueno & 3 & 0 & 0 & 3 & \\
\hline & Total & & 7 & 17 & 26 & 50 & \\
\hline
\end{tabular}

* Prueba de McNemar-Bowker.

Fuente: elaboración propia 


\section{Discusión}

Actualmente las actividades de proyección social impartidas desde el ámbito universitario representan una de las estrategias más importantes para impactar a la comunidad. En odontología, los niños en edad escolar representan una población vulnerable que requiere continua intervención para prevenir desde temprana edad patologías de alta prevalencia como la caries dental y enfermedad gingival. Si bien, los estudiantes universitarios se valen de una serie de estrategias (charlas, videos, carteleras, macromodelos dentales) para realizar enseñanza en salud oral, ya que la aceptación y apropiación de conocimientos en niños de corta edad es un reto. La falta de atención, los distractores y desinterés son algunos de los obstáculos que enfrentan quienes intentan transmitir la información. Esta situación incentiva la búsqueda de nuevas estrategias que capten la atención de los niños y permitan un proceso de aprendizaje (11).

Bernal-Álvarez T et al., exponen que el uso de las TICs (presentaciones interactivas con diapositivas, videos, guía interactiva, avatar entre otros), puede arrojar resultados positivos en aprendizajes y conocimientos de hábitos de higiene bucal, siempre y cuando estas sean constantes y motivadoras. El objetivo de la educación se logra siempre y cuando se empleen los métodos apropiados, teniendo en cuenta las características de cada grupo, particularmente cuando se trabaja con población infantil (12).

González R et al. (13), compararon dos programas educativos y su influencia en la salud bucal de preescolares. Concluyeron que el programa educativo en salud bucal "Sonrisa feliz" obtuvo mejores resultados que el programa tradicional expositivo. Puesto que dentro las metodologías de este programa se incluyeron demostraciones, actividades de refuerzo, juegos y el sistema de economía de fichas que lograron motivar al preescolar en el cuidado de su salud bucal y tuvo mayor aceptación tanto en preescolares como en sus padres, demostrando la relevancia del uso de diversas metodologías lúdicas en los programas de salud bucal dirigidos a los niños.

Si bien ya existen cómics o historietas previamente concebidos desde una perspectiva didáctica; se tiene conocimiento de trabajos en los que se utiliza el lenguaje del cómic para transmitir contenidos que de otro modo para los niños serían difíciles de aprender. Cuando las historietas cumplen con las condiciones de buena legibilidad en los aspectos físico, lingüístico, psicológico y conceptual, se pueden constituir en un recurso didáctico que provoca una respuesta favorable resultando útil tanto para los niños como para los padres $(14,15)$. 
Desde este punto de vista, el aporte fundamental de este trabajo fue demostrar el uso de la historieta específicamente como una herramienta para la educación en salud oral. Los resultados demuestran que existe asimilación de los conceptos e ideas de salud oral por parte de los niños después de interactuar con la historieta, estos conocimientos a su vez se vieron reflejados en la disminución del índice de biopelícula lograda por la aplicación de los conocimientos adquiridos en cuanto a cepillado, uso de seda y enjuague bucal. Es necesario resaltar que los niños de 6 a 7 años, aunque no dominaban 100 \% la lectura en el momento de implementar la historieta, siguieron la secuencia de imágenes obteniendo excelentes y buenos resultados en el postest. Esto los respalda Nava Borrero (8) quien concluyó que los comics o historietas sirven como documentos complementarios para el estudio de temas específicos, en este caso se utilizaron para transmitir conocimientos de salud oral que son más asimilables si el niño tiene una fuente de representación de imágenes como la ofrece el comics.

Las razones que respaldan la eficacia de la historieta como herramienta para la educación llegándose a implementar en distintas asignaturas y áreas del conocimiento incluyendo salud oral, se basan en las características y ventajas propias que ofrece esta estrategia de comunicación. El lenguaje del cómic permite a los niños el aprendizaje de secuencias de imágenes, la potenciación de su imaginación, así como el desarrollo de la socialización mientras realizan una actividad lúdica como es la lectura de historietas, de esta manera favorece la concentración y captación de la atención de niños y niñas promoviendo de manera didáctica el aprendizaje (16-18).

\section{Conclusiones}

Los resultados de esta investigación demuestran que la historieta resultó ser un medio educativo práctico y efectivo al momento de trabajar con población infantil. Al incluirla como componente lúdico durante la enseñanza en salud oral se logró un incremento en el conocimiento y asimilación de conceptos en comparación con el método tradicional de charla y demostración.

\section{Referencias}

1. Colombia. Ministerio de Salud. IV Estudio nacional de salud bucal - ENSAB [Internet]. Disponible en: IV.https://www.minsalud.gov.co/sites/rid/Lists/BibliotecaDigital/RIDE/VS/ PP/ENSAB-IV-Situacion-Bucal-Actual.pdf. 
2. Stein C, Lopes-Santos N, Balbinot-Hilgert J, Neves-Hugo F. Effectiveness of oral health education on oral hygiene and dental caries in schoolchildren: Systematic review and meta-analysis. Community Dent Oral Epidemiol. 2018; 46(1): 30-37. doi: 10.1111/cdoe.12325.

3. Sukumaran A, Pradeep A. Early Childhood Caries: Prevalence, Risk Factors, and Prevention. Front Pediatr. 2017; 5:1-7. doi: 10.3389/fped.2017.00157.

4. Tan-Suárez NT, Montes-de Oca C, Tan-Suárez N. Educación Comunitaria en Salud Bucal para niños. Rev Hum Med. 2003; 3(2).

5. Sanabria-Vázquez D, Ferreira-Gaona M, Pérez-Bejarano N, Díaz-Reissner C, Torres-Amarilla C, Cubilla M, etal. Caries dental en menores en situación de pobreza, asistidos por una fundación en Paraguay. Rev. Estomatol. Herediana. 2016; 26(2): 70-77. doi: https://doi.org/10.20453/reh. V26i2.2868.

6. John BJ, Asokan S, Shankar S. Evaluation of different health education interventions among preschoolers: a randomized controlled pilot trial. J Indian Soc Pedod Prev Dent. 2013; 31(2): 96-9. doi: 10.4103/0970-4388.115705.

7. Nazri-Yanti G, Mayasari-Alamsyah R, Ella-Natassa S. Effectiveness of dental health education using cartoons video showing method on knowledge and oral hygiene of deaf children in Yayasan Karya Murni Medan. Int J Appl Dent Sci. 2017; 3(2): 86-90.

8. Lazarinis F, Mazaraki A, Verykios V, Panagiotakopoulos, C. E-comics in teaching: Evaluating and using comic strip creator tools for educational purposes. In 2015 10th International Conference on Computer Science \& Education (ICCSE). p. 305-309. doi: 10.1109/ICCSE.2015.7250261.

9. Eker C, Karadeniz O. The Effects of Educational Practice with Cartoons on Learning Outcomes. Int J Humanit Soc Sci. 2014; 4(14): 223-34.

10. Van-Wyk MM. The use of cartoons as a teaching tool to enhance student learning in economics education. J Soc Sci. 2011; 26(2): 117-30. doi: https://doi.org/10.1080/09718923.2011.11 892888.

11. Inocente-Díaz, ME, Pachas-Barrionuevo, FdM. Educación para la Salud en Odontología. Revista Estomatológica Herediana.2012; 22(4): 232-241. doi: https://doi.org/10.20453/reh. V22i4.91. 
12. Bernal-Álvarez T, Santos-Machado YP, Alzate-Yepes T, Morales-Mora ME, Agudelo-Suárez AA, Posada-López A, Martínez-Londoño A. Las nuevas tic y los Métodos Educativos Tradicionales (met) en la educación para la salud bucal en estudiantes de Básica Primaria. Rev Nac Odontol. 2014; 10(19): 15-22. doi: http://dx.doi.org/10.16925/od.v10i19.842

13. González-Chávez R, García-Rupaya C. Comparación de dos programas educativos y su influencia en la salud bucal de preescolares. Kiru. 2013; 10(1): 18-25.

14. Tatalovic M. Science comics as tools for science education and communication: A brief, exploratory study. J Sci Commun. 2009; 8(4): 1-17.

15. Cheesman K. Using comics in the science classroom. J Coll Sci Teach. 2006; 48-52.

16. Barbosa A, Tavares G, Kruta A. The comics as teaching strategy in learning of students in an undergraduate management program. Rev. Adm. Mackenzie. 2017; 18(1): 40-65. doi: http://dx.doi.org/10.1590/1678-69712017/administracao.v18n1p40-65.

17. García-Cantó E, Cuadrado-Ruíz J, Amor-García M, Argudo-Ramírez M. El cómic como recurso didáctico para el aprendizaje de las actividades físicas en el medio natural en el $2^{\circ}$ ciclo de la educación primaria española REXE. Revista de Estudios y Experiencias en Educación. 2010; 9(17): 117-133. Disponible en: http://www.redalyc.org/articulo.oa?id=243116388007

18. Gabaron S. The power of print reading: comics in the classroom. J Cell Commun Signal. 2017; 11(3): 285-290. doi: 10.1007/s12079-017-0400-z. 